\title{
Effectiveness of A Package of Folate-oriented Tertiary Interventions on Incidence of Fetus and Birth Defects: protocol for a single blind cluster randomized controlled trial
}

\section{Yi Zhang}

Children's Hospital of Fudan University

\section{Mengru Li}

Children's Hospital of Fudan University

\section{Xiaotian Chen}

Children's Hospital of Fudan University

\section{Dingmei Wang}

Children's Hospital of Fudan University

Mi Ji

Children's Hospital of Fudan University

\section{Yuan Jiang}

Children's Hospital of Fudan University

\section{Yalan Dou}

Children's Hospital of Fudan University

\section{Xiaojing Ma}

Children's Hospital of Fudan University

\section{Wei Sheng}

Children's Hospital of Fudan University

\section{Guoying Huang}

Children's Hospital of Fudan University

Weili Yan ( $\nabla$ yanwl@fudan.edu.cn)

Children's Hospital of Fudan University

\section{Study protocol}

Keywords: Serum folate, Red blood cell folate, Birth defects, Periconception health care, Cluster randomized controlled trial, Study protocol

Posted Date: May 9th, 2019 
DOI: https://doi.org/10.21203/rs.2.9453/v1

License: (c) (1) This work is licensed under a Creative Commons Attribution 4.0 International License. Read Full License 


\section{Abstract}

Background: Birth defects are the main cause of fetal death, infant mortality and morbidity, as well as long-term disability in China. Though the etiology of birth defects remains unknown, many studies indicated that maternal key nutrition factors, especially maternal folate status during periconception period, played an important role in organogenesis, the incidence of neural tube defects, congenital heart diseases and some other birth defects. The aim of this trial is to evaluate the effectiveness of package of folate-oriented interventions during periconception on incidence reduction of fetus and birth defects. Methods: This is a two-arm single-blind cluster randomized controlled trial (CRCT) in clinical settings. Twenty-two tertiary community health care centers will be randomly allocated to the intervention arm or control arm. Eligible women will be recruited before conception and receive folate-oriented tertiary intervention procedures in addition to the routine perinatal care (intervention arm), or only routine perinatal care (control arm) according to the national recommendation and guideline. The difference was the procedures to aware and ensure sufficient folate levels before conception in the intervention arm. The participants will be blinded to the allocation. Participants of the control arm will not be offered any information about the intervention procedures during the study period to prevent contamination. The composite primary outcome is defined as occurrence of fetus defects, still birth, and neonatal birth defects identified from the confirmation of pregnancy to 28 days after birth. Secondary outcomes include abortions, financial burden of fetus and birth defects, gestational diabetes, gestational hypertension, pregnancy weight gain (entire and early gestation), maternal body weight and $\mathrm{HbA} 1 \mathrm{c}$ at and after 6 months of delivery and neonatal development. Discussion: This trial will provide important evidence on the effectiveness of a package of folate-oriented tertiary interventions on the reduction of the incidence of fetus and birth defects compared to routine periconceptional and antenatal health care. This evidence is expected to impact policy making about improving primary prevention of birth defects in China. Trial registration: ClinicalTrials.gov, NCT03725878区Registered on 24 October 2018, https://www.clinicaltrials.gov/ct2/show/NCT03725878?term=Huang+guoying\&rank=2

\section{Background}

Birth defects, also known as congenital anomalies or congenital malformations, are defined as structural or functional abnormalities, affecting on physical, intellectual, and social wellbeing [1]. Birth defects are a diversity group of disorders occurred during intrauterine and existed before and after birth, including neural tube defects, congenital palate and cleft lip, malformations of genital organs, deformations of the musculoskeletal system and metabolic disorders, et al. According to data from World Health Organization (WHO), more than 8 million (a total of $6 \%$ births) are born with severe birth defects each year and nearly 303000 newborn deaths worldwide are caused by birth defects [2]. Also, an estimation that at least 3.3 million of children aged 0 to 5 years died of severe birth defects and approximately 3.2 million of children with disabilities related to birth defects every year [2, 3]. In China, where over $5.6 \%$ of live births present with a birth defect [4]. And studies show that incidence of birth defects were more than 10\% [5]. Congenital heart defects, oral clefts, hydrocephalus, neural tube defects and mental retardation are the 
most common birth defects in China, which is similar to that of worldwide. Birth defects are a lifethreatening cause leading to neonatal death, still birth, and long-term disability, which may significantly influence individuals' physical and mental health, families, health-care systems, and societies. The etiology of birth defects remain unknown though inheritance factors, environmental teratogens or micronutrient malnutrition have been widely studied. Among them, maternal key nutrition factors, especially maternal folic acid status arouse intense concern.

Folate is a water-soluble vitamin abounded in green vegetables and fruits. Whereas folic acid is a synthetic form of folate, and its bioavailability is approximately $70 \%$ higher than that of folate [6]. Commonly, folate status in population is accessed by measuring serum or red blood cell folate (RBC folate) through biochemistry methods. And a cut-off point to define folate deficiency is serum folate 3 $\mathrm{ng} / \mathrm{mL}(6.8 \mathrm{nmol} / \mathrm{L})$ or RBC folate $<100 \mathrm{ng} / \mathrm{mL}(226.5 \mathrm{nmol} / \mathrm{L})$ recommended by WHO (using macrocytic anemia as a haematological indicator) [7]. Folate is a key nutrition factor involved in multiple cellular processes, such as nucleotide synthesis, DNA repair, genomic stability, mitochondrial protein synthesis and methylation reactions including histone methylation. Folic acid supplementation can decrease the occurrence of several types of congenital abnormalities, which has been widely studied. Neural tube defects (NTDs) account for one of the most prevalent group of birth defects, which is a severe structural defect resulting from the failed closure of the neural tube during early embryogenesis period. Studies showed prevalence of NTDs was significantly reduced with the supplementary of folic acid ADDIN EN.CITE [8-11] . And Khoshnood appeals that voluntary fortification of folic acid failed to reduce NTDs effectively, thus policies for mandatory fortification of food staples with folic acid should be issued to prevent NTDs ADDIN EN.CITE $[11,12]$. RBC folate $>400 \mathrm{ng} / \mathrm{mL}(906 \mathrm{nmol} / \mathrm{L})$ is recommended to prevent NTDs by WHO [13]. Populations based studies showed strong evidences on folate reducing occurrence of congenital heart disease (CHD). A Hungarian study showed approximately $40 \%$ of CHDs may be reduced by periconceptional folic acid-containing multivitamin supplementation [14]. And a reduction of prevalence of $\mathrm{CHD}$ subtypes, such as conotruncal defects and coarctation of the aorta, was founded to be strongly relevant to periconceptional food fortification with folic acid ADDIN EN.CITE [15]. The protective effect on folic acid supplementation reducing other outcomes have also been observed, such as cleft lip and palate, miscarriages or other birth defects ADDIN EN.CITE $[16,17]$. However, association between folic acid and cleft lip was largely inconclusive for lacking Randomized controlled trials (RCTs) evidences [18].

Investigation on the correlation between single nucleotide polymorphisms (SNPs) of folate metabolism pathway genes with susceptibility to birth defects has been widely studied. The C677T polymorphism of the 5,10-methylenetetrahydrofolate reductase (MTHFR) gene may reduce the MTHFR enzyme activity, which lower maternal folate status and increase the susceptibility of NTDs and CHDs ADDIN EN.CITE [19, 20] . However, a meta-analysis showed no relation between C677T polymorphism and CHD risks ADDIN EN.CITE [21] . Chinese researchers found SNPs in folate metabolism pathway genes (MTR, CBS, MTRR and FIGN) may increase risk of CHDs via different mechanisms ADDIN EN.CITE [22-25] . Routine pregnant health cares, including NTDs, Down's syndrome screening, and ultrasound examination are used 
worldwide to detect chromosomal abnormalities and major structural malformations ADDIN EN.CITE [26]

Although importance of sufficient folate nutrition during periconception period to fetal development is widely known, prepregnant folate supplementation population is limited even in developed countries ADDIN EN.CITE [27-30] , and folate deficiency affects about half pregnant women in China [31]. We aim to conduct a randomized intervention trial to test the effectiveness and feasibility of a package of folateoriented tertiary interventions on incidence and clinical outcomes of birth defects. The experimental intervention includes pre-, during pregnancy and after birth, especially emphasis on ensuring sufficient RBC folate levels before pregnancy.

\section{Aims and objectives}

The aim of this trial is to evaluate the effectiveness of the monitoring and insurance of sufficient folic acid supplement during periconception on incidence reduction of fetus and birth defects.

Based on these objectives, the primary hypothesis is that the intervention group receiving a package of folate-oriented tertiary interventions will have lower incidence of fetus and birth defects than the control group. The secondary hypotheses are that the incidence rate of total abortions because of the affected congenital defects; incidence of death or severe organ dysfunctions; the cost that relates to affecting any birth defects during pregnancy and after birth will reduce by the tertiary intervention; and the metabolic and development-related outcomes of mothers and children have improved.

\section{Methods}

\section{Design}

This protocol refers to the SPIRIT 2013 (Standard Protocol Items: Recommendations for Interventional Trials) Statement. The participant timeline is presented in Fig 1. A SPIRIT checklist is given in additional file 1.

This is a two-arm single-blind cluster randomized controlled trial (CRCT) in clinical settings.

\section{Participants}

Study population is female who prepares for pregnancy. Participants meet the follow criteria are eligible to this study: (1) females are planning for pregnant within a year; (2) women are aged 18 to 45 years old. There are no exclusion criteria. Eligible women are recruited as candidate participants at pre-pregnancy physical examinations clinics or premarital health checkup centers in Minhang district and Songjiang district in Shanghai (see Table S1). A written informed consent is obtained from each candidate participants at recruitment. Among them, only candidate participants finally get pregnant during study period will be a valid participant and be included as study subjects for outcome analysis. 


\section{Sample size and randomization}

Sample size calculation will be performed based on the primary outcome: the incidence of a composite rate of fetus defects, still birth, or birth defects. The proportion of primary endpoint was conservatively estimated to be $8 \%$ in the control arm according two observational studies [5,32], and $4 \%$ in the intervention arm (or $50 \%$ reduction) based on a meta-analysis of randomized intervention trials of folic acid supplementation intervention in folate-deficient population [33]. With cluster size of 100 subjects per center, $\mathrm{K}$ coefficient of 0.23 , a power of $85 \%$ and a $5 \%$ level of type I error, 22 clusters (2200 pregnant women or 1100 in each arm) will be required. To allow for a $10 \%$ dropout rate, at least 110 pregnant women will be recruited in each center.

Permuted block randomisation method with block size of 4 will be used. Stata 15 was used for randomisation code generation. Twenty-two community health care centres (clusters) will be allocated to the intervention and control arms. Randomisation list will be generated by an independent statistician.

\section{Procedures}

Following random allocation, eligible subjects will receive tertiary intervention procedures plus the routine perinatal care (intervention arm), or only routine perinatal care (control arm) according to the national recommendation and guidelines [34]. The women in both arms will receive the baseline measurements. For the intervention arm, a package of preconception intervention procedures will be conducted. After the baseline measurements, once the participant from both arms get pregnant, they will receive prenatal intervention procedures and will be followed throughout the entire pregnancy. After delivery, a 6-months follow up will be conducted to collect clinical outcomes among infants. In addition, the routine clinical data including participants and their babies will be extracted from local maternal clinic antenatal electronic medical record system. The flow chart of the CRCT procedure is depicted in Fig. 2. The overview of the biomarkers throughout the study are summarized in Table 1.

Comprehensive strategies are used to retain participants in the study. A smartphone APP were provided to each community health care center to help identify and follow the cohort subjects timely. In addition, an automated text message system is adopted to remind participants of schedule and appointment of follow-up.

\section{Outcomes}

The primary endpoint is defined as occurrence of fetus defects, still birth, and neonatal birth defects identified from the confirmation of pregnancy to 28 days after birth. This is a composite outcome: fetus defects detected by Down's syndrome screenings, Nuchal translucency (NT) examinations and Ultrasound image examinations during the second trimester; stillbirth; and the number of birth defects after delivery diagnosed by clinical team. In consistent with the national birth defects surveillance policy, a total of 24 types of defects were defined (details of defects types see Table S2). In addition, the pregnancy suspension including miscarriages and artificial abortions will be recorded. Detailed 
information of abortions will be collected, including time of abortion, reasons for abortion, and antenatal information before abortion.

The major secondary outcomes include: (1) the proportion (\%) of total abortions that related to congenital defects. (Time Frame: from the confirmation of pregnant to the 28th gestational week); (2) the incidence (\%) of infant death or severe organ dysfunctions (composite outcome) (Time Frame: from birth to 6 months after delivery (can be expanding to the end of the 7th month)); (3) extra medical cost that related to fetus and birth defects during pregnancy and after birth (Time Frame: from confirmation of pregnancy to one year old after birth).

In addition, we also included the following outcomes: the incidence of neonatal congenital heart defects, maternal gestational diabetes (\%), gestational hypertension (\%), pregnancy weight gain $(\mathrm{kg})$ (entire and early gestation), maternal body weight $(\mathrm{kg})$ and $\mathrm{HbA} 1 \mathrm{c}(\%)$ at and after 6 months of delivery and neonatal development as secondary outcomes. neonatal development will be evaluated by the infant body weight (g) at birth, 42day, 3 months and 6 months.

\section{Measurements}

\section{The nutrient supplement questionnaire}

Two questionnaires will be used for nutrient supplement data collection. Questionnaire 1 will be administered at recruitment before pregnancy to collect information on folic acid supplement, vitamin supplement, the brand and content of nutrient supplement, maternal education, socio-demographic status, occupation, smoking status, alcohol consumption, BMI, any medication use, health status. Questionnaire 2 will be used at the early pregnancy. Besides the information in Questionnaire 1, use of drug information, reproductive history and health status were also included (Please see Appendix 2).

\section{Collection of blood samples}

We will keep the rest of fasting serum and EDTA anticoagulation blood samples of peripheral venous blood after routine laboratory examination. The fasting serum will be divided into three light-proof EP tubes within six hours, as well as EDTA anticoagulation blood samples. Divided samples will be stored in -20 degrees freezers temporarily at clinical settings and transported by three trained investigators to the central biobank for storage in -80 degrees freezers within two weeks. The protocol for blood sample tagging, isolation, temporary storage, transportation and permanent storage see Appendix 3.

\section{Nutrient evaluation \& genotyping of key enzymes}

EDTA anticoagulation blood will be collected to measure RBC folate and serum folate, homocysteine, vitamin $\mathrm{D}$, vitamin $\mathrm{B} 12$ and ferritin assays. All six biomarkers are performed on an electrochemiluminescence assay (ARCHITECT i2000SR Analyzer, Abbott Laboratories, USA). A standard solution with known concentration (produced by Abbott Laboratories) will be used to quality control every day before the examination. If the level of quality control were out of the range of concentration, the 
examination would be suspended and adjusted. External quality control is carried out with the control lab data programme from Abbott Laboratories (Abbott Laboratories, Shanghai, China). RBC folate concentrations will be adjusted using hematocritif the RBC folate concentration is below $126.0 \mathrm{ng} / \mathrm{ml}$ or above $651.1 \mathrm{ng} / \mathrm{ml}$, meanwhile, the serum folate level is over $3.5 \mathrm{ng} / \mathrm{ml}$. The hematocrit data will be extracted from the hospital laboratory information system. Those examinations will be performed in central laboratory of Children's Hospital of Fudan University.

The serum concentration of vitamin A and vitamin E will be quantitatively detected by liquid chromatography-tandem mass spectrometry in central laboratory of Children's Hospital of Fudan University. The testing instrument is triple quadrupole mass spectrometer LC/MS/MS System (NO: API 3200MDTM, ABSciex Pte.Ltd.). A standard solution of vitamin A-d6 and vitamin E-d6 are applied as internal standards.

We also plan to examine other blood chemicals in the future using the stored serum including cholesterol, high-density lipoprotein, low-density lipoprotein, triglyceride, fasting glucose and heavy metals. Cholesterol, high-density lipoprotein, low-density lipoprotein, triglyceride, and fasting glucose were performed on Beckman coulter AU chemistry analyzers (Beckman, USA) in central laboratory of Children's Hospital of Fudan University. Serum heavy metals including Mg, Fe, Zn, Se, Mn, As, Cu and Ca, will be analyzed by inductively coupled plasma mass spectrometry (ICP-MS) (Inductively Coupled Plasma Optical iCAP6300, Themo ${ }^{\circledR}$, USA) in a standard mode. The metals examination will be conducted in Instrumental Analysis Center of Shanghai Jiaotong University.

The SNPs involved in folate metabolism pathway will be genotyped in subjects with unknown low RBC folate concentration, including BHMT (rs3733890), CBS (rs2850144, rs2851391), FIGN (rs2119289), MTHFD1 (rs2236225), MTHFR (rs1801131, rs1801133, rs3737965), MRT (rs1805087, rs28372871, rs1131450), MTRR (rs1801394, rs326119), RFC1 (rs1051266), and SHMT (rs1979277) ADDIN EN.CITE $[25,35-39]$. Genotyping will be performed using the TaqMan allelic discrimination assay in 96-well plates on the platform of 7500 Real-time polymerase chain reaction (PCR) System (Applied Biosystems, Foster City, CA).

\section{Interventions}

\section{The control arm}

The participant will only receive routine perinatal care according to the national guidelines [34], including premarital health checkup, pre-pregnancy physical examinations, the antenatal care, NT examination, Down's screening, chromosomal abnormalities and major structural malformations screened by ultrasound examination, and neonatal health care.

\section{The intervention arm}

In addition to the routine perinatal care as described in the control arm, the participants in the intervention arm will receive a package of folate-oriented tertiary intervention procedures, which includes 
preconception procedures and pregnant \& neonatal procedures. The details are as follow:

(i) Preconception procedures:

We examine the participant serum and RBC folate concentrations to identify subjects with folate deficiency (defined as RBC folate concentration $<400 \mathrm{ng} / \mathrm{ml}$ ). By reviewing of subjects' self-administered questionnaire, and folate metabolism ability evaluated by genotyping key genomic variants, a phone counseling will be conducted to provide an individualized folic acid supplementary guide. The goal of the counseling is to ensure their RBC folate concentration $>400 \mathrm{ng} / \mathrm{ml}$ before conception by sufficient dose and length of folate supplementation.

(ii) Pregnant \& Neonatal procedures

Fetus and infants with birth defect(s) will be referred via a green channel to an authorized tertiary obstetric hospital and pediatric hospital respectively to receive further routine comprehensive assessments for prenatal diagnosis. The parents are referred to a multi-discipline pediatrics team for a sufficient consultation about possible postnatal treatments in the future if the pregnancy remains to avoid unnecessary abortions. All pregnancies will be followed up at delivery to help clinical team for early diagnosis, treatment and related procedures. All infants from the two arms will continuously be followed up till 6 months after birth to collect data of clinical outcomes. This green channel is also provided to the subjects from the control arm.

\section{Masking}

The study is single blinded. The study investigators who are involved in recruitment, the laboratory staff, and the dose-adjusting investigator (who was not involved in participant care) will remain blinded. The investigator involved in intervention and the statistician was not blinded, as unbinding was required in order to carry out statistical procedures.

All researchers conducting outcome assessments will be masked in the trial. The trial statistician will also be blinded regarding the treatment code when he develops the statistical analysis plan and writes the statistical programmes, which will be validated and completed using dummy randomization codes. The actual allocation will only be provided to the study team after lock of the database.

\section{Data management and analyses}

STATA 15.0 will be employed to perform all statistical analyses. All quantitative data will be collected using paper questionnaires with unique ID numbers for each recruited patient. Data will be stored at the research team office at the end of each day. Daily checking of data will be carried out by the research coordinator, with queries identified and resolved promptly. A database will be built using Access 2013 (Microsoft office Professional Plus 2013); double entry and checking will be performed by an assigned data entry team. Discrepancies will be resolved by a third data manager. Once in an electronic file, all data 
will be password protected, with data managers controlling access to passwords and ensuring the database is backed up daily.

Findings of the trail will be reported according to the CONSORT guidelines for CRCT. The primary outcome is a binary composite outcome. A formal statistical analysis will be performed as a two-sided test, using chi-square test. The treatment effect will be estimated as the difference of the rate in the control arm minus that in the experimental arm (\%) and $95 \%$ Cls. In the primary analysis, participants lost to follow-up will be included in the denominators but will not be considered as positive events in the numerator.

We identified clinical and healthcare factors likely to influence both the probability of receiving intervention and outcome based on the scientific literature and biological plausibility. The factors include cluster, block, actual cluster size, age, BMI (body mass index)ఐpregnant history (pregnant time and miscarriage) $₫$ and exposure to smoking (mother or father's smoking status, yes/no) at baseline. This will be done to correct for any possible baseline imbalances between the arms after randomization.

Generalised estimating equation (GEE) model will be performed to estimate risk ratio and $95 \% \mathrm{Cl}$, as well as risk difference and $95 \% \mathrm{Cl}$, by adjusting cluster with link of identity. The above log-binomial GEE model may not converge when all covariates are introduced into the model simultaneously. If this occurs, a covariate will be removed from log-binomial GEE model until GEE model converge.

Subgroup analyses will be performed on age, BMI, adverse pregnant history, exposure to smoking, RBC folate level at baseline. Age will be categorized at $<35$ years or more; BMI will be dichotomized at 24 $\mathrm{kg} / \mathrm{m} 2$; RBC folate level will be cut off at $400 \mathrm{ng} / \mathrm{ml}$ before pregnancy. Assessment of the homogeneity of treatment effect by a subgroup variable will be conducted by a GEE with the treatment, subgroup variable, and their interaction term as predictors, center as cluster, and the P-value presented for the interaction term. In addition, the risk ratio of the primary endpoint will be derived from the GEE model with binomial distribution function, and log link function, respectively.

All secondary outcomes will be analyzed as a superiority design and two-sided $95 \% \mathrm{Cls}$ for the treatment differences in these outcomes between the two arms will be calculated and presented. Secondary outcome analyses will be based on the intention-to-treat (ITT) population unless specified.

Binary secondary outcomes will be analyzed with the same strategy and method with that is used for the primary outcome. Generalized estimating equation (GEE) model will be performed with treatment as the only predictor, center as cluster effect using binomial distribution and log link function, from which the treatment effect will be estimated as the risk ratio between intervention and the control arm together with $95 \% \mathrm{Cls}$. If the above log-binomial regression model does not converge, GEE model with normal distribution and log link function will be used.

The continuous outcome will be summarized using number of subjects (n), mean, standard deviation (SD), minimum, and maximum by intervention group, and will be analyzed by a General liner model (GLM) model with treatment as fixed effect and with normal distribution and identity link function. Difference in mean outcome with their two-sided $95 \%$ confidence intervals between two groups will be 
derived from the GLM model. For repeated measurement variables, such as baby body weight at different visit, or maternal body weight during gestation, will be analyzed using mixed effect linear regression model, with treatment, visit, interaction between treatment and visit as fixed effects, baseline measurement as covariate, cluster and subject as random effects.

We calculate the total cost of each subject including cost of treatment, nursing, and rehabilitation from recruitment to the follow-up endpoint. The differences of total cost will be compared between the two arms using the same methods described above by treating which as a continuous variable. The total cost of intervention arm included folic acid supplements and RBC folate examinations before conception. All the cost is settled in RMB. Further comprehensive cost-effectiveness analysis will be described by a separated SAP.

All analyses will be described in detail in the finalized and signed statistical analysis plan before unmasking the study.

\section{Study management}

A research management office will be set up for this study to monitor the process and verify protocol compliance once a month. The management office is responsible for data management, biological sample management, quality control, and participant management (decision to terminate the trial). Data correctness will be assessed and serious adverse events will be reported to the ethics committee of the particular center and Children's Hospital of Fudan University. The ethics committee of Children's Hospital of Fudan University has the authority to terminate the trial according to local laws or institutional regulations. The investigators should keep each care center, ethics committee, and the journal informed of any major protocol modifications.

\section{Ethical approval}

The trial protocol received ethical approval from Ethic board of Children's Hospital clinical pharmacology IRB of Fudan University (Approval Number: 2018-151). Written informed consent will be obtained from each recruited participant before the questionnaire survey and intervention.

\section{Discussion}

This trial will examine the effectiveness of standard tertiary interventions on the reduction of the incidence of fetus and birth defects compared to routine health care. The intervention has several advantages over routine health care: (i) Pre-pregnancy interventions are an individualized, comprehensive intervention program that references the questionnaire, genes, and nutrient levels in the blood; (ii) This intervention study builds a complete healthcare network from pre-pregnancy to childbirth, from community to hospital; (iii) It may improve self-management of health in primary prevention of birth defects, because participants will receive individualized interventions and follow-up health instructions from pre-pregnancy to childbirth in community settings. Even in the case of null results, this trial will 
produce a large amount of illuminating data for perinatal health care. If the interventions are effective, it will provide an additional health care option for comprehensive health management of reduction of birth defects.

\section{Trial status}

The Protocol Version is 1.0. The trial is registered at ClinicalTrials.gov with identifier number NCT03725878.

Recruitment started on October 31, 2018 and the study is anticipated to be completed in December, 2021.

\section{Abbreviations}

BMI: Body mass index; CHD: Congenital heart disease; GEE: Generalised estimating equation; GLM: General liner model; ITT: Intention-to-treat; MTHFR: The 5, 10-methylenetetrahydrofolate reductase; NT: Nuchal translucency; NTDs: Neural tube defects; PCR: Polymerase chain reaction; RBC: Red blood cell; RCTs: Randomized controlled trials; SNPs: Single nucleotide polymorphisms.

\section{Declarations}

\section{Acknowledgements}

We are extremely grateful to all the families who take part in this study, the doctors from community health care centers who conduct recruitment, and the whole study team that includes interviewers, computer and laboratory technicians, clerical workers, research scientists, volunteers, managers, receptionists and nurses.

\section{Funding}

This work is supported by National key research and development program (Grant No: 2016YFC1000506), Natural Science Foundation of China (Grant No: 81273168) and the Three-year Planning for Strengthening the Construction of Public Health System in Shanghai (GWIV-24).

\section{Availability of data and materials}

Not applicable.

\section{Authors' contributions}

Substantial contributions to the conception or design of the work were made by $G H$ and $W Y$. $X C, Y Z, M L$, YJ, DW, MJ, YD, XM, WS and WY contributed to the development of the protocol. WY, YZ, XC made the statistical analysis plan, in charge of data management, and will perform the analysis. WY, ZY and ML drafted the work. The interpretation of data for the work was performed by WY, GH, YZ, JY, DW, ML and 
XC. All authors revised the work critically for important intellectual content, and gave final approval of the version to be published.

\section{Ethical approval}

The trial protocol received ethical approval from Ethic board of Children's Hospital clinical pharmacology IRB of Fudan University (Approval Number: 2018-151). Written informed consent will be obtained from each recruited patient before the intervention and questionnaire survey. We will not begin recruiting at other centres in the trial until local ethical approval has been obtained.

Consent for publication

Not applicable

Conflict of interest

The authors have no conflicts of interest.

\section{Reference}

1. Sixty-Third World Health Assembly WHO: Human organ and tissue transplantation. Resolution WHA 63.22 of the Sixty-Third World Health Assembly. Cell Tissue Bank2010, 11(4):411-412.

2. [http://www.who.int/news-room/fact-sheets/detail/congenital-anomalies]

3. [https://www.who.int/en/news-room/fact-sheets/detail/children-reducing-mortality]

4. Health CMo: China Birth Defect Prevention Report (2012). In.; 2012.

5. Xuelei Yin XD, Mingzhu Yao: Analysis on the composition of 481 neonates with congenital malformation and their associated factors. $j$ Clin Pediatr 2008, 26(3):204-208.

6. Proceedings of the Joint Nutrition Symposium of the European Society of Veterinary and Comperative Nutrition, the European College of Veterinary and Comperative Nutrition, the American Academy of Veterinary Nutrition, the American College of Veterinary Nutrition, the European Zoo Nutrition Research Group, and the Comperative Nutrition Society. August 21-25, 2002. Antwerp, Belgium. J Anim Physiol Anim Nutr (Berl) 2004, 88(3-4):73-177.

7. Organization WH: Serum and red blood cell folate concentrations for assessing folate status in populations, vol. Vol. WHO/NMH/NHD/EPG/15.01; 2015.

8. Czeizel AE, Dudas I: Prevention of the first occurrence of neural-tube defects by periconceptional vitamin supplementation. N Engl J Med 1992, 327(26):1832-1835. 
9. Wang H, De Steur H, Chen G, Zhang X, Pei L, Gellynck X, Zheng X: Effectiveness of Folic Acid Fortified Flour for Prevention of Neural Tube Defects in a High Risk Region. Nutrients 2016, 8(3):152.

10. Williams J, Mai CT, Mulinare J, Isenburg J, Flood TJ, Ethen M, Frohnert B, Kirby RS, Centers for Disease C, Prevention: Updated estimates of neural tube defects prevented by mandatory folic Acid fortification - United States, 1995-2011. MMWR Morb Mortal Wkly Rep 2015, 64(1):1-5.

11. De-Regil LM, Fernandez-Gaxiola AC, Dowswell T, Pena-Rosas JP: Effects and safety of periconceptional folate supplementation for preventing birth defects. Cochrane Database Syst Rev 2010(10):CD007950.

12. Khoshnood B, Loane M, de Walle H, Arriola L, Addor MC, Barisic I, Beres J, Bianchi F, Dias C, Draper E et al: Long term trends in prevalence of neural tube defects in Europe: population based study. BMJ 2015, 351:h5949.

13. Cordero AM, Crider KS, Rogers LM, Cannon MJ, Berry RJ: Optimal serum and red blood cell folate concentrations in women of reproductive age for prevention of neural tube defects: World Health Organization guidelines. MMWR Morb Mortal Wkly Rep 2015, 64(15):421-423.

14. Czeizel AE, Dudas I, Vereczkey A, Banhidy F: Folate deficiency and folic acid supplementation: the prevention of neural-tube defects and congenital heart defects. Nutrients 2013, 5(11):4760-4775.

15. Liu S, Joseph KS, Luo W, Leon JA, Lisonkova S, Van den Hof M, Evans J, Lim K, Little J, Sauve R et al: Effect of Folic Acid Food Fortification in Canada on Congenital Heart Disease Subtypes. Circulation 2016, 134(9):647-655.

16. Wehby GL, Murray JC: Folic acid and orofacial clefts: a review of the evidence. Oral Dis 2010, 16(1):11-19.

17. Li S, Chao A, Li Z, Moore CA, Liu YH, Zhu JH, Erickson JD, Hao L, Berry RJ: Folic Acid Use and Nonsyndromic Orofacial Clefts in China A Prospective Cohort Study. Epidemiology 2012, 23(3):423-432.

18. Czeizel AE: Is folic acid a risk factor for oral clefts? Eur J Epidemio/ 2013, 28(11):841-843.

19. Frosst P, Blom HJ, Milos R, Goyette P, Sheppard CA, Matthews RG, Boers GJH, Denheijer M, Kluijtmans LAJ, Vandenheuvel LP et al: A Candidate Genetic Risk Factor for Vascular-Disease - a Common Mutation in Methylenetetrahydrofolate Reductase. Nat Genet 1995, 10(1):111-113.

20. Shaw GM, Rozen R, Finnell RH, Wasserman CR, Lammer EJ: Maternal vitamin use, genetic variation of infant methylenetetrahydrofolate reductase, and risk for spina bifida. American Journal of Epidemiology 1998, 148(1):30-37.

21. Mamasoula C, Prentice RR, Pierscionek T, Pangilinan F, Mills JL, Druschel C, Pass K, Russell MW, Hall D, Topf A et al: Association Between C677T Polymorphism of Methylene Tetrahydrofolate Reductase and 
Congenital Heart Disease: Meta-Analysis of 7697 Cases and 13125 Controls. Circ-Cardiovasc Gene 2013, 6(4):347-353.

22. Zhao JY, Qiao B, Duan WY, Gong XH, Peng QQ, Jiang SS, Lu CQ, Chen YJ, Shen HB, Huang GY et al: Genetic variants reducing MTR gene expression increase the risk of congenital heart disease in Han Chinese populations. Eur Heart J 2014, 35(11):733-742.

23. Zhao JY, Yang XY, Gong XH, Gu ZY, Duan WY, Wang J, Ye ZZ, Shen HB, Shi KH, Hou J et al: Functional Variant in Methionine Synthase Reductase Intron-1 Significantly Increases the Risk of Congenital Heart Disease in the Han Chinese Population. Circulation 2012, 125(3):482-+.

24. Zhao JY, Yang XY, Shi KH, Sun SN, Hou J, Ye ZZ, Wang J, Duan WY, Qiao B, Chen YJ et al: A functional variant in the cystathionine beta-synthase gene promoter significantly reduces congenital heart disease susceptibility in a Han Chinese population. Cell Res 2013, 23(2):242-253.

25. Wang DA, Wang F, Shi KH, Tao H, Li Y, Zhao R, Lu H, Duan WY, Qiao B, Zhao SM et al: Lower Circulating Folate Induced by a Fidgetin Intronic Variant Is Associated With Reduced Congenital Heart Disease Susceptibility. Circulation 2017, 135(18):1733-+.

26. Rydberg C, Tunon K: Detection of fetal abnormalities by second-trimester ultrasound screening in a non-selected population. Acta Obstet Gyn Scan 2017, 96(2):176-182.

27. Nilsen RM, Leoncini E, Gastaldi P, Allegri V, Agostino R, Faravelli F, Ferrazzoli F, Finale E, Ghirri P, Scarano $\mathrm{G}$ et al: Prevalence and determinants of preconception folic acid use: an Italian multicenter survey. Ital J Pediatr 2016, 42(1):65.

28. Wang Dingmei ZY, Ye Ying, Jiang Yuan, Ma Xiao-jing, Yan Wei-li, Huang Guo-ying: A cross-sectional study on prevalence of paternal and parental preconception folic acid supplement use and its influencing factors. Chinese Journal of Evidence-based Pediatrics. 12(1) 2017:1-6.

29. Cueto HT, Riis AH, Hatch EE, Wise LA, Rothman KJ, Mikkelsen EM: Predictors of preconceptional folic acid or multivitamin supplement use: a cross-sectional study of Danish pregnancy planners. Clin Epidemio/ 2012, 4:259-265.

30. Hoyo C, Murtha AP, Schildkraut JM, Forman MR, Calingaert B, Demark-Wahnefried W, Kurtzberg J, Jirtle RL, Murphy SK: Folic acid supplementation before and during pregnancy in the Newborn Epigenetics STudy (NEST). BMC Public Health 2011, 11(1):46.

31. Liu Yan CY, Sun Xiao, Wang Jinling, Liu Qilan: Analysis on the dietary survey results of preconceptional couples in Liyang city, Jiangsu province. MATERNAL \& CHILD HEALTH CARE OF CHINA 2011, 2011, 26(21).

32. Lixin Zheng ML, Qiling Wang, Shanshan Xu, Ganhua Pan, Jiangchu Huang, Meizhen Zeng: Investigation on birth defects of fetus after twelve weeks pregnancy and children with 0-5 yares old in 
part area of Guangdong prevince chin J Fam Plann 2017, 25(2):93-97.

33. Blencowe $\mathrm{H}$, Cousens $\mathrm{S}$, Modell B, Lawn J: Folic acid to reduce neonatal mortality from neural tube disorders. Int J Epidemiol 2010, 39 Suppl 1:i110-121.

34. Measures for the implementation of the law of the People's Republic of China on maternal and infant health care [http://www.chinawch.org.cn/zfcg/flfg/200809/t20080917_49941.html]

35. Heil SG, Van der Put NMJ, Waas ET, den Heijer M, Trijbels FJM, Blom HJ: Is mutated serine hydroxymethyltransferase (SHMT) involved in the etiology of neural tube defects? Mol Genet Metab 2001, 73(2):164-172.

36. Chango A, Emery-Fillon N, de Courcy GP, Lambert D, Pfister M, Rosenblatt DS, Nicolas JP: A polymorphism (80G-> A) in the reduced folate carrier gene and its associations with folate status and homocysteinemia. Mol Genet Metab 2000, 70(4):310-315.

37. Huang T, Tucker KI, Lee YC, Crott JW, Parnell LD, Shen JA, Smith CE, Ordovas JM, Li D, Lai CQ: Methylenetetrahydrofolate Reductase Variants Associated with Hypertension and Cardiovascular Disease Interact with Dietary Polyunsaturated Fatty Acids to Modulate Plasma Homocysteine in Puerto Rican Adults. Journal of Nutrition 2011, 141(4):654-659.

38. Saraswathy KN, Asghar M, Samtani R, Murry B, Mondal PR, Ghosh PK, Sachdeva MP: Spectrum of MTHFR gene SNPs C677T and A1298C: a study among 23 population groups of India. Mol Biol Rep 2012, 39(4):5025-5031.

39. Field MS, Kamynina E, Agunloye OC, Liebenthal RP, Lamarre SG, Brosnan ME, Brosnan JT, Stover PJ: Nuclear Enrichment of Folate Cofactors and Methylenetetrahydrofolate Dehydrogenase 1 (MTHFD1) Protect de Novo Thymidylate Biosynthesis during Folate Deficiency. J Biol Chem 2014, 289(43):2964229650.

\section{Tables}

Table 1 Parental biomarkers evaluated in the ETC study 


\begin{tabular}{|c|c|c|c|}
\hline Biomarkers & Sample type & $\begin{array}{l}\text { Baseline } \\
\text { (Male and female) }\end{array}$ & $\begin{array}{l}\text { Before } 13 \text { weeks } \\
\text { (Female) }\end{array}$ \\
\hline Folate, $\mathrm{ng} / \mathrm{mL}$ & Serum & $x$ & \\
\hline RBC Folate, $\mathrm{ng} / \mathrm{mL}$ & Whole blood & $x$ & \\
\hline Homocysteine, $\mu \mathrm{mol} / \mathrm{L}$ & Serum & $x$ & \\
\hline Vitamin $\mathrm{D}, \mathrm{ng} / \mathrm{mL}$ & Serum & $x$ & \\
\hline Vitamin B12, pg/mL & Serum & $x$ & \\
\hline Vitamin $\mathrm{A}, \mu \mathrm{g} / \mathrm{mL}$ & Serum & $x$ & \\
\hline Vitamin $\mathrm{E}, \mu \mathrm{g} / \mathrm{mL}$ & Serum & $x$ & \\
\hline Iron protein, $\mathrm{ng} / \mathrm{mL}$ & Serum & $x$ & \\
\hline Metals (Fe, Zn, Se, Cu, Ca, ect) ,mg/L & Serum & $x$ & \\
\hline DNA & Whole blood & $x$ & $x$ \\
\hline Genotyping & DNA & $x$ & \\
\hline $\mathrm{CHOL}, \mathrm{HDL}, \mathrm{LDL}, \mathrm{TG}$, Fasting glucose & Serum & $x$ & \\
\hline
\end{tabular}

\section{Figures}




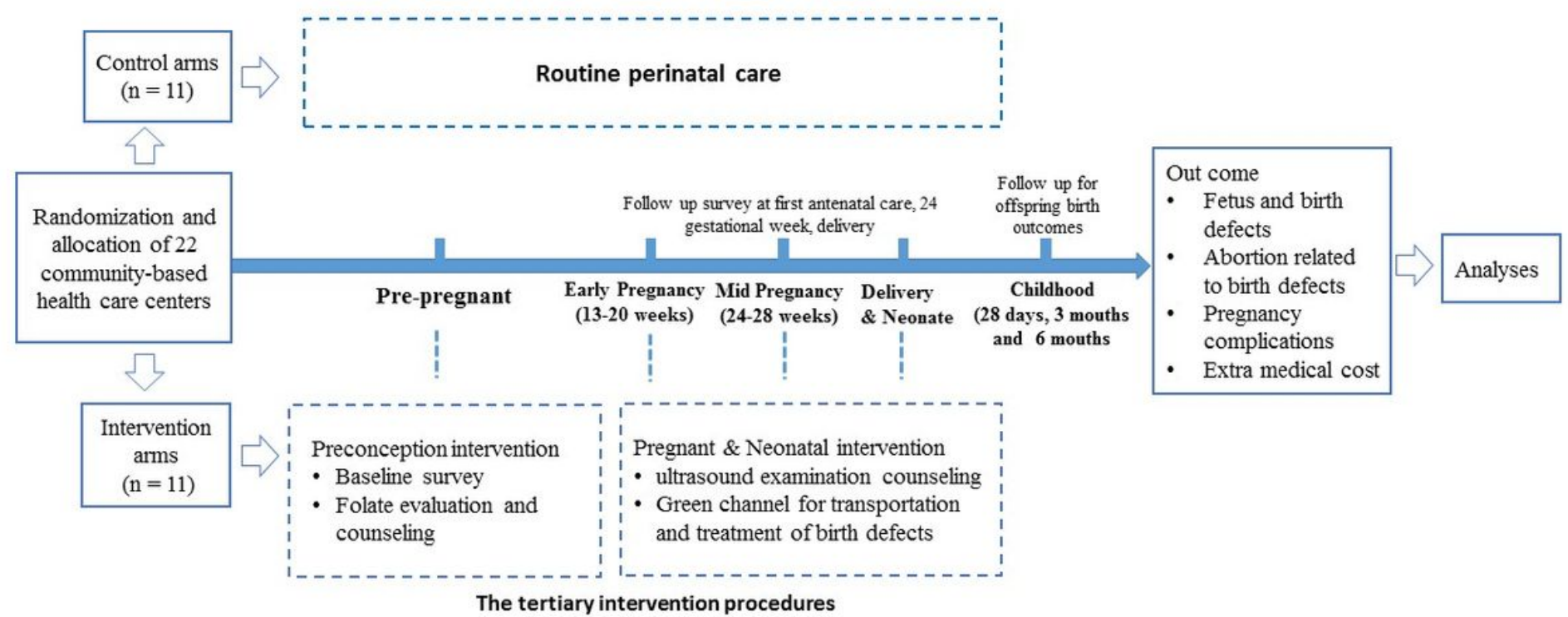

Figure 1

Example template of recommended content for the schedule of enrolment, interventions, and assessments.*

\section{Supplementary Files}

This is a list of supplementary files associated with this preprint. Click to download.

- TableS2Typesoffetusdefectsandbirthdefects.docx

- TableS1siteslist.docx

- SPIRITFillablechecklistfinal.doc 